\title{
Tunable Metal Oxide Frameworks via Coordination Assembly of Preyssler- Type Molecular Clusters
}

\author{
Linfeng Chen, Khin A. San, Michael J. Turo, Milan Gembicky, Shelir Fereidouni, Mark Kalaj \\ and Alina M. Schimpf* \\ Department of Chemistry and Biochemistry, University of California, San Diego, La Jolla, CA \\ 92093, USA \\ *Electronic address: aschimpf@ucsd.edu
}

Supplementary Tables and Figures...................................................S2

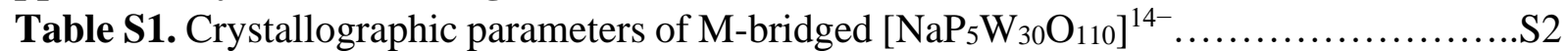

Figure S1. Structure depicting octahedral coordination of bridging metal ions...............S3

Figure S2. Powder X-ray diffraction of Co frameworks before and after $\mathrm{N}_{2}$ sorption.........S3

Figure S3. Conversion of $\mathrm{Co}^{2+}$-bridged frameworks to octahedral coordination..............S4

Figure S4. X-ray photoelectron spectrum of Fe-bridged frameworks....................S5

Figure S5. Oxidation of as-synthesized Zn-bridged frameworks .........................S6

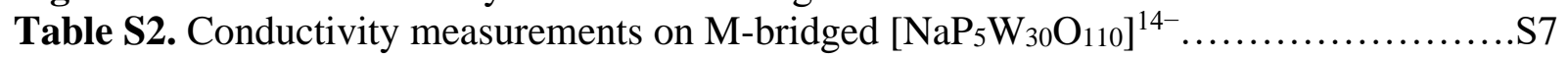

Figure S6. SEM-EDS mapping of mixed-metal frameworks........................S7

Figure S7. High-magnification mapping of finely powdered mixed-metal frameworks.......S8

Figure S8. Powder X-ray diffraction patterns of mixed-metal frameworks..................S9

Table S3. Nominal and experimental ratios in mixed-metal frameworks..................S9

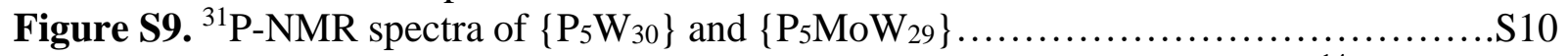

Table S4. Crystallographic parameters of $\mathrm{Co}$ - and Fe-bridged $\left[\mathrm{NaP}_{5} \mathrm{MoW}_{29} \mathrm{O}_{110}\right]^{14-}{ }^{1 . . . . . . . . . S 10}$

Figure S10. Powder X-ray diffraction patterns of Mo-doped frameworks................S11

Table S5. Conductivity measurements on Mo-doped frameworks.........................S11

Figure S11. X-ray photoelectron spectra of Mo-doped frameworks......................S12

Figure S12. ${ }^{31} \mathrm{P}$ NMR spectrum of cavity-exchanged clusters...........................S12

Table S6. Crystallographic parameters of Co-bridged $\left[\mathrm{ZP}_{5} \mathrm{MoW}_{29} \mathrm{O}_{110}\right]^{12-}(\mathrm{Z}=\mathrm{Sm}, \mathrm{Bi}) \ldots . . \mathrm{S} 13$

Table S7. Cluster connections in Co-bridged $\left[\mathrm{ZP}_{5} \mathrm{MoW}_{29} \mathrm{O}_{110}\right]^{12-}(\mathrm{Z}=\mathrm{Bi}, \mathrm{Sm}) \ldots \ldots \ldots \ldots . . . \mathrm{S} 14$

Experimental Methods................................................................S14

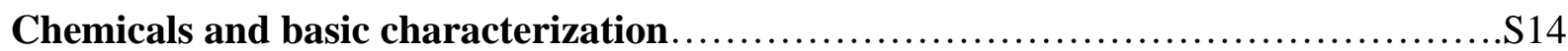

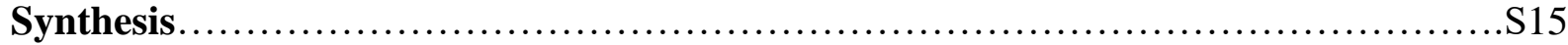

Structural determination...................................................... 18

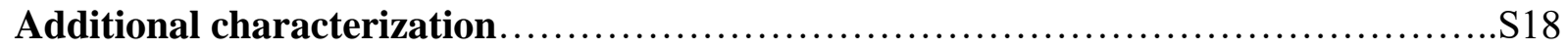

References...............................................................................S19 


\section{Supplementary Tables and Figures}

Table S1. Crystallographic parameters of M-bridged $\left[\mathrm{NaP}_{5} \mathrm{~W}_{30} \mathrm{O}_{110}\right]^{14-}$

\begin{tabular}{|c|c|c|c|c|c|}
\hline Bridge & Mn & $\mathbf{F e}$ & $\mathbf{N i}$ & $\mathbf{C u}$ & $\mathbf{Z n}$ \\
\hline $\begin{array}{l}\text { Empirical } \\
\text { formula }\end{array}$ & $\begin{array}{c}\mathrm{Mn}_{3.3} \mathrm{~K}_{1.7} \mathrm{Li}_{0.3} \\
\mathrm{Na}_{2.4} \mathrm{O}_{125} \mathrm{P}_{5} \mathrm{~W}_{30}\end{array}$ & $\begin{array}{c}\mathrm{Fe}_{3.5} \mathrm{~K}_{2.8} \mathrm{LiNa}_{1.3} \\
\mathrm{O}_{128.3} \mathrm{P}_{5} \mathrm{~W}_{30}\end{array}$ & $\begin{array}{c}\mathrm{Ni}_{3} \mathrm{~K}_{1.3} \mathrm{Li}_{0.6} \mathrm{Na}_{3} \\
\mathrm{O}_{133.7} \mathrm{P}_{5} \mathrm{~W}_{30}\end{array}$ & $\begin{array}{c}\mathrm{Cu}_{3} \mathrm{~K}_{3.3} \mathrm{Na}_{1.5} \\
\mathrm{O}_{133.5} \mathrm{P}_{5} \mathrm{~W}_{30}\end{array}$ & $\begin{array}{c}\mathrm{Zn}_{3.5} \mathrm{~K}_{3.3} \mathrm{Na}_{1.6} \\
\mathrm{O}_{138.6} \mathrm{P}_{5} \mathrm{~W}_{30}\end{array}$ \\
\hline $\begin{array}{l}\text { Formula } \\
\text { weight }\end{array}$ & 7977.09 & 8062.37 & 8074.85 & 8159.28 & 8280.28 \\
\hline $\begin{array}{l}\text { Crystal size } \\
\left(\mathrm{mm}^{3}\right)\end{array}$ & $\begin{array}{c}0.055 \times 0.041 \times \\
0.024\end{array}$ & $\begin{array}{c}0.048 \times 0.035 \times \\
0.031\end{array}$ & $\begin{array}{c}0.074 \times 0.052 \times \\
0.031\end{array}$ & $\begin{array}{c}0.062 \times 0.041 \\
\times 0.018\end{array}$ & $\begin{array}{c}0.042 \times 0.027 \\
\times 0.024\end{array}$ \\
\hline$a(\AA)$ & $52.303(3)$ & $52.178(3)$ & $52.101(9)$ & $52.548(3)$ & $52.137(3)$ \\
\hline$b(\AA)$ & 21.664(2) & $21.6024(12)$ & $21.545(4)$ & $21.6349(12)$ & $21.5598(12)$ \\
\hline$c(\AA)$ & $26.055(3)$ & $26.1423(16)$ & $26.104(5)$ & $25.8226(16)$ & $26.1562(16)$ \\
\hline $\begin{array}{l}\text { Volume } \\
\left(\AA^{3}\right)\end{array}$ & $29522(6)$ & 29467(3) & 29302(9) & $29357(3)$ & 29401(3) \\
\hline$Z$ & 8 & 8 & 8 & 8 & 8 \\
\hline$\rho_{\text {calc }}\left(\mathrm{g} / \mathrm{cm}^{3}\right)$ & 3.589 & 3.635 & 3.661 & 3.692 & 3.741 \\
\hline$\mu\left(\mathrm{mm}^{-1}\right)$ & 23.743 & 23.877 & 24.020 & 24.081 & 24.175 \\
\hline $\begin{array}{l}\text { Goodness- } \\
\text { of-fit on } \mathrm{F}^{2}\end{array}$ & 1.094 & 1.050 & 1.070 & 1.051 & 1.059 \\
\hline $\begin{array}{l}\text { Reflections } \\
\text { collected }\end{array}$ & 100879 & 81170 & 118263 & 116493 & 114520 \\
\hline $\begin{array}{l}\text { Independent } \\
\text { reflections }\end{array}$ & 13581 & 16100 & 15037 & 15619 & 15064 \\
\hline$R_{1}$ & 0.0679 & 0.0722 & 0.0864 & 0.0881 & 0.0887 \\
\hline$w R$ & 0.1607 & 0.1579 & 0.1811 & 0.2074 & 0.2113 \\
\hline $\begin{array}{l}\text { Average } \mathrm{M}- \\
\mathrm{O}_{\text {cluster }} \\
\text { distance }(\AA)\end{array}$ & 2.09 & 2.05 & 2.01 & 2.06 & 2.04 \\
\hline CSD \# & 1955439 & 1955438 & 1955437 & 1955440 & 1955441 \\
\hline
\end{tabular}

Radiation: $\operatorname{MoK}_{\alpha}(\lambda=0.71073)$

Temperature: $100 \mathrm{~K}$

Crystal system: orthorhombic

Space group: Imma 


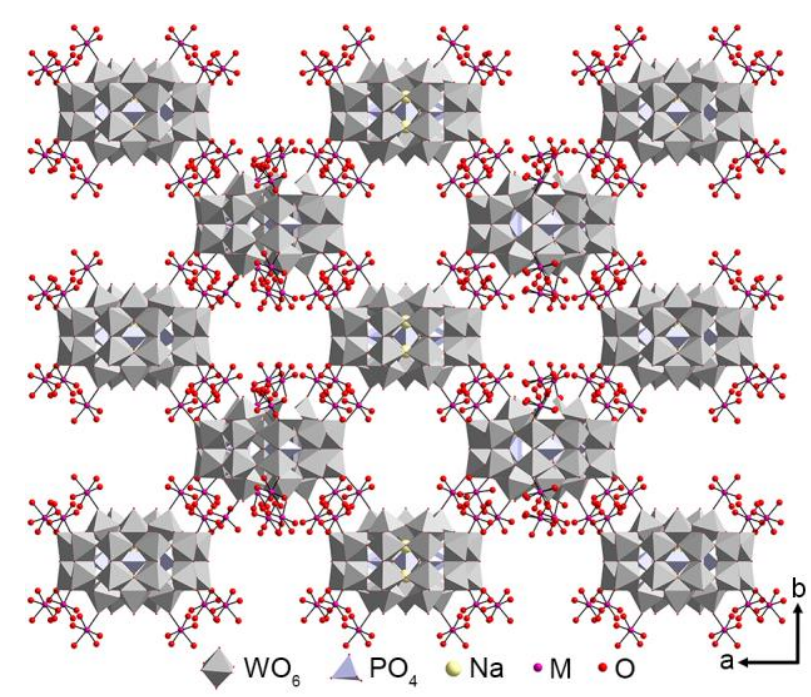

Figure S1. Structural representation of $\left[\mathrm{Co}\left(\mathrm{H}_{2} \mathrm{O}\right)_{4}\right]^{2+}$-bridged frameworks depicting octahedral coordination of the bridging metal ions.

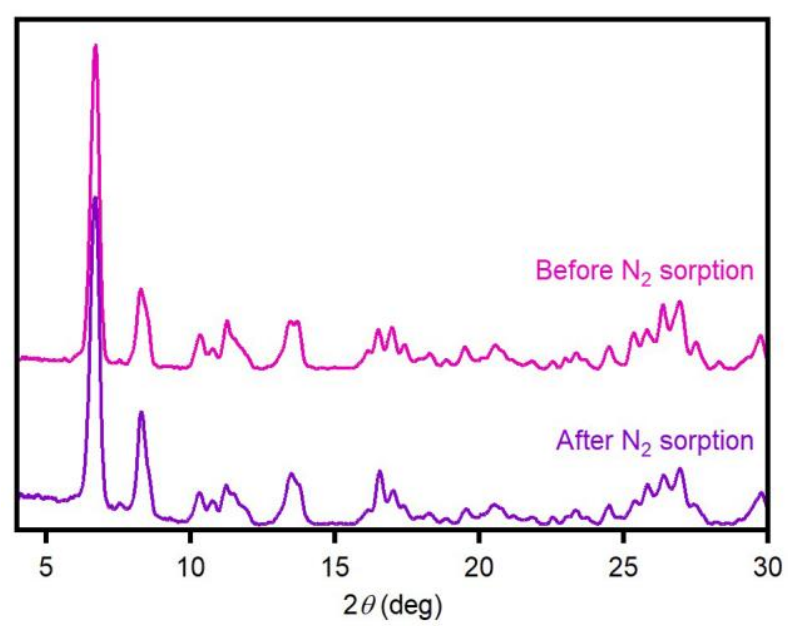

Figure S2. Powder X-ray diffraction patterns of $\left[\mathrm{Co}\left(\mathrm{H}_{2} \mathrm{O}\right)_{4}\right]^{2+}$-bridged frameworks before (top) and after (bottom) $\mathrm{N}_{2}$ sorption. The powder pattern is different from those shown in Figure 1 due to changes upon removal from the mother liquor. ${ }^{1}$ 

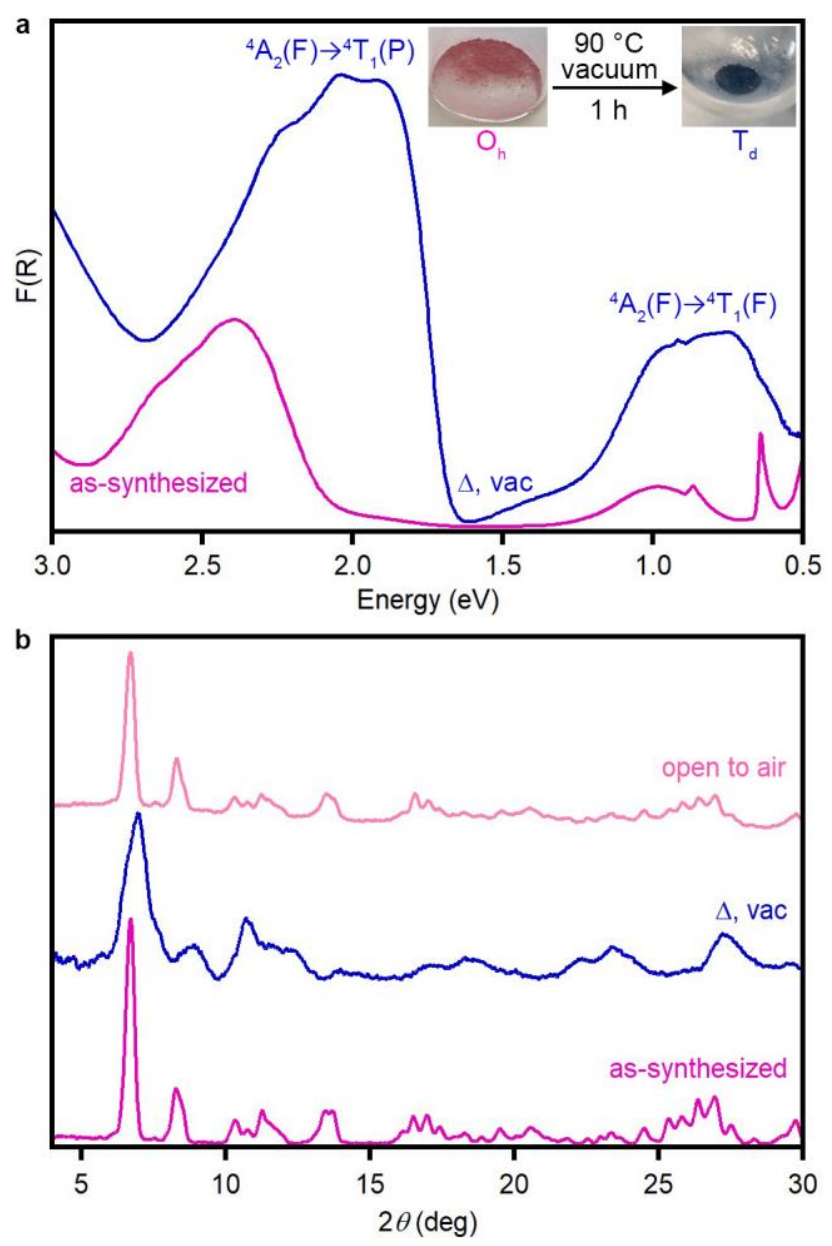

Figure S3. (a) Diffuse reflectance spectra of $\mathrm{Co}^{2+}$-bridged frameworks before (bottom, pink) and after (top, blue) heating under vacuum at $90{ }^{\circ} \mathrm{C}$ for $1 \mathrm{~h}$, showing conversion of $\mathrm{Co}^{2+}$ from octahedral to tetrahedral coordination. Inset: Photographs showing color change. (b) Powder X-ray diffraction patterns of the same frameworks reveal a change in the crystal structure (middle) compared to the original powders (bottom), which is recovered upon exposure of the tetrahedral frameworks to air (top). The powder pattern is different from those shown in Figure 1 due to changes upon removal from the mother liquor. ${ }^{1}$ 


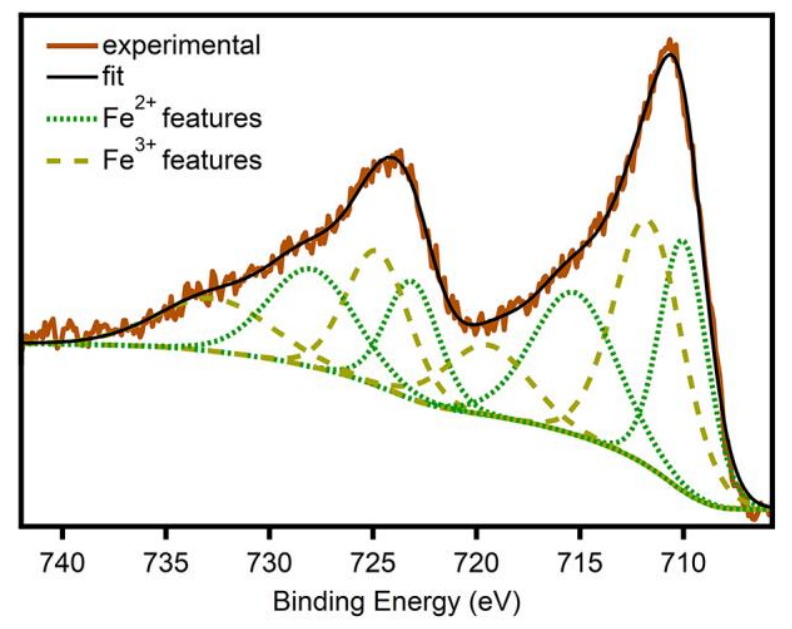

Figure S4. Fe $2 p$ X-ray photoelectron spectrum of Fe-bridged frameworks. 


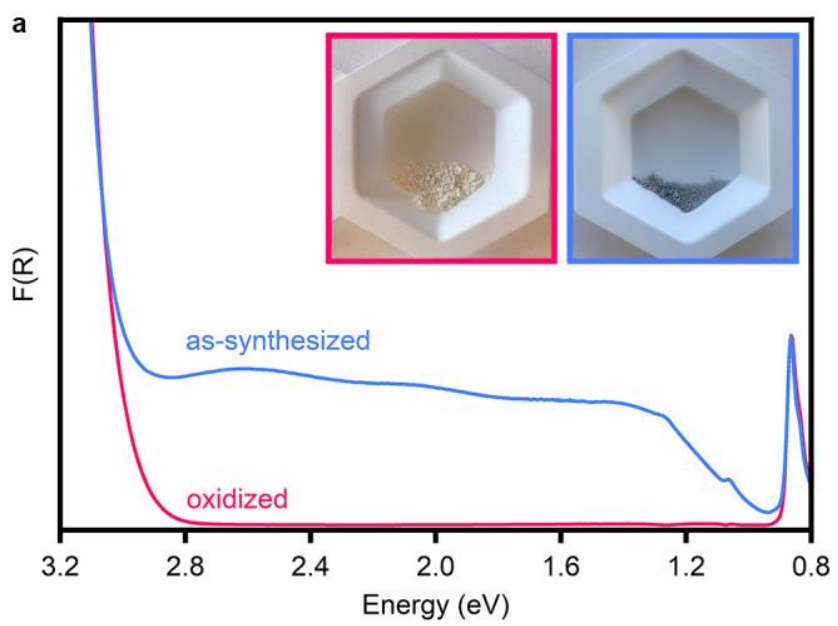

b
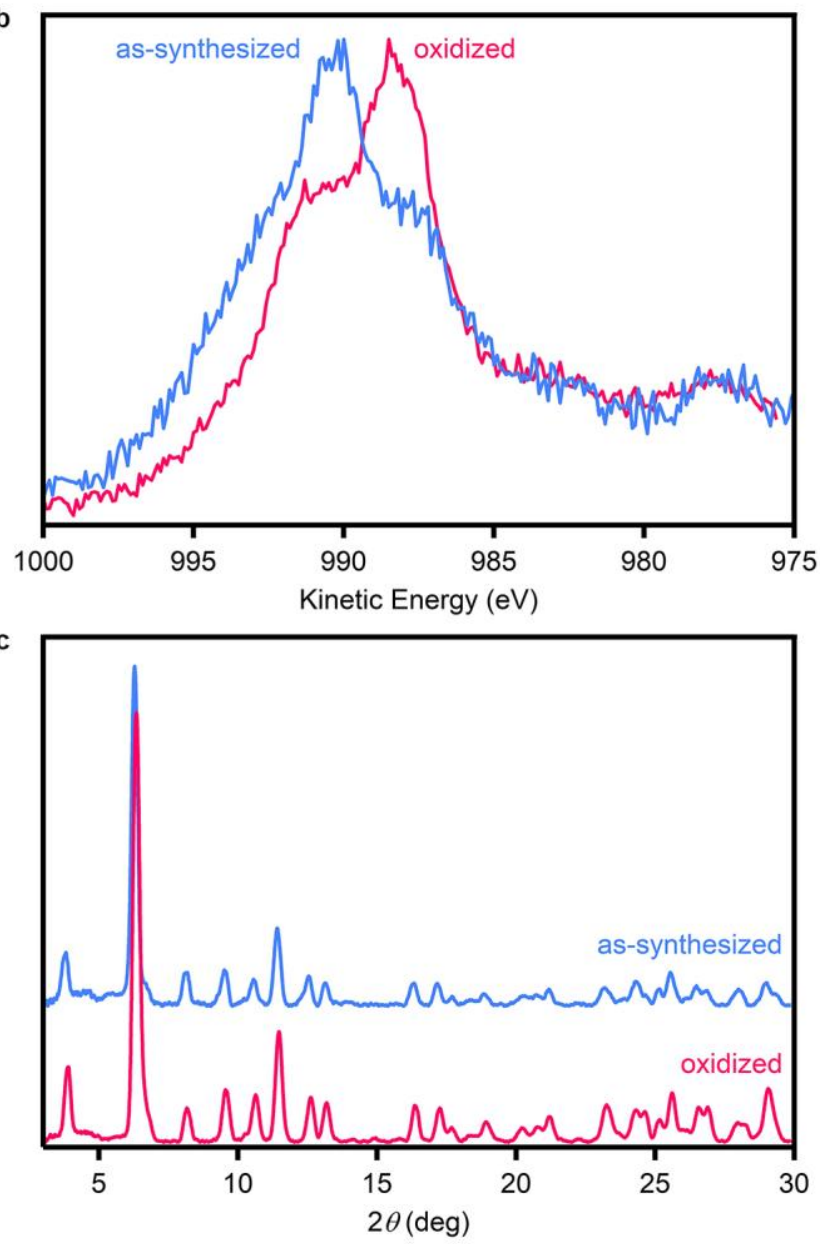

Figure S5. (a) Diffuse reflectance spectra (b) Zn LMM X-ray photoelectron spectra and (c) powder X-ray diffraction patterns and of Zn-bridged frameworks as-synthesized (blue) and after oxidation with $\mathrm{H}_{2} \mathrm{O}_{2}$ (red). Inset: Photographs showing the color of the frameworks before (blue, right) and after (left, red) oxidation. The loss of gray color and the shift in the LMM X-ray photoelectron spectrum are indicative of a change from $\mathrm{Zn}^{0}$ to $\mathrm{Zn}^{2+}$ upon oxidation, ${ }^{2-3}$ while the powder X-ray diffraction patterns show no disruption of the framework structure. 
Table S2. Individual conductivities $(\mu \mathrm{S} / \mathrm{cm})$ of metal-bridged frameworks and unbridged $\left\{\mathrm{P}_{5} \mathrm{~W}_{30}\right\}$ clusters. Numbers in parentheses are the \% humidity at which each measurement was performed.

$\begin{array}{rccccccc} & (51 \%) & (52 \%) & (54 \%) & (48 \%) & (48 \%) & (40 \%) & (49 \%) \\ \text { Average } & \mathbf{2 . 8} & \mathbf{1 5} & \mathbf{5} & \mathbf{2 . 0} & \mathbf{3 . 5} & \mathbf{2 . 0 5} & \mathbf{0 . 3} \\ \text { Standard deviation } & 0.9 & 2 & 1 & 0.7 & 0.9 & 0.09 & 0.2\end{array}$

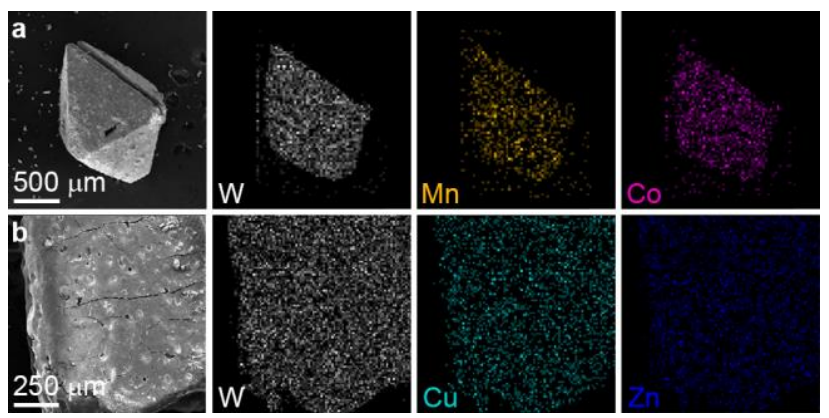

Figure S6. SEM-EDS mapping of frameworks with (a) $0.5 \mathrm{Mn} / \mathrm{Co}$ and (b) 1.4 $\mathrm{Cu} / \mathrm{Zn}$ mixed-metal bridging-units. 


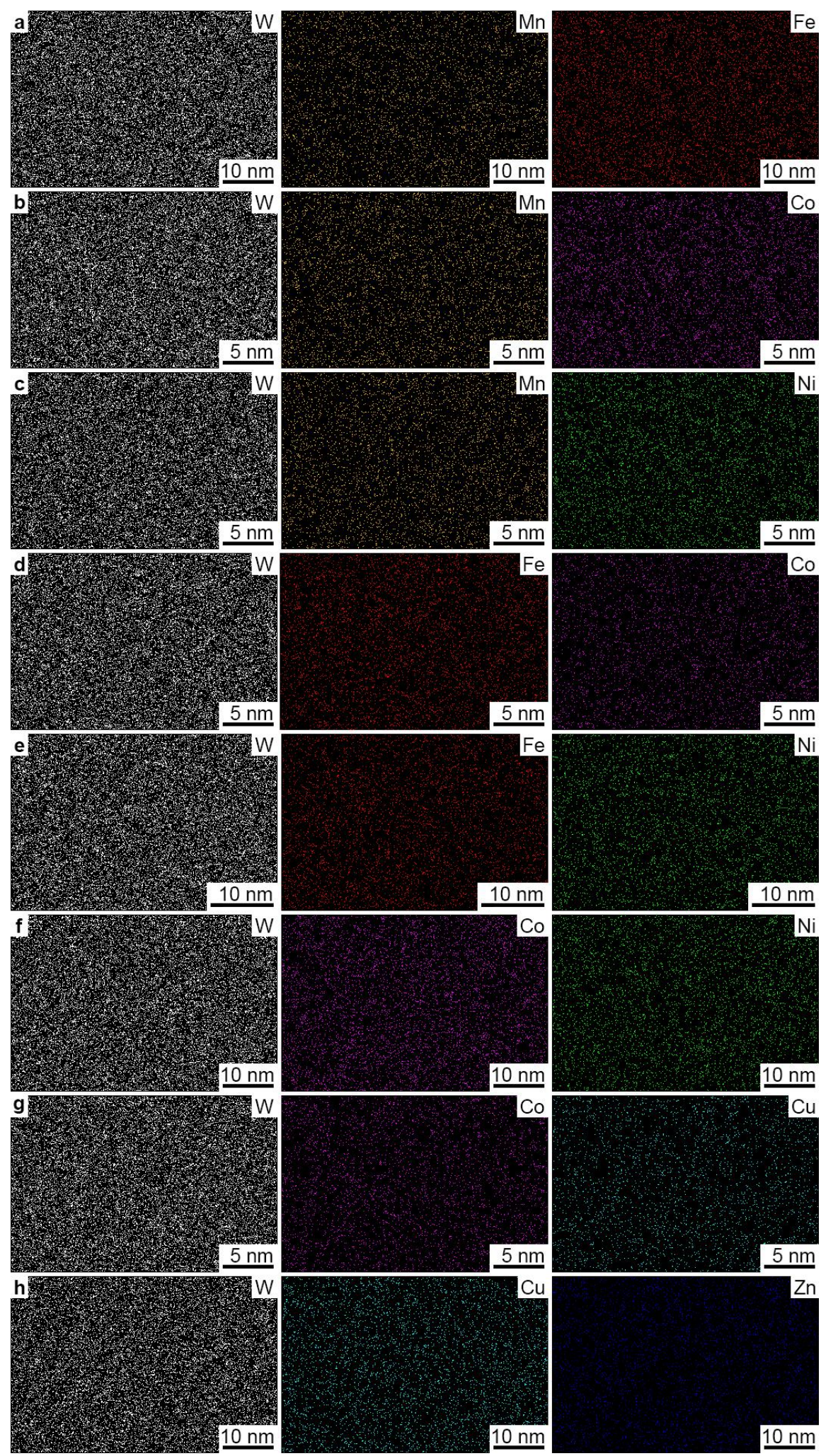

Figure S7. SEM-EDS mapping of frameworks with (a) $0.5 \mathrm{Mn} / \mathrm{Fe}$, (b) $0.5 \mathrm{Mn} / \mathrm{Co}$, (c) $0.4 \mathrm{Mn} / \mathrm{Ni}$, (d) $1.0 \mathrm{Fe} / \mathrm{Co}$, (e) $1.0 \mathrm{Fe} / \mathrm{Ni}$, (f) $1.1 \mathrm{Co} / \mathrm{Ni}$, (g) $1.7 \mathrm{Co} / \mathrm{Cu}$ and (h) $1.4 \mathrm{Cu} / \mathrm{Zn}$. 


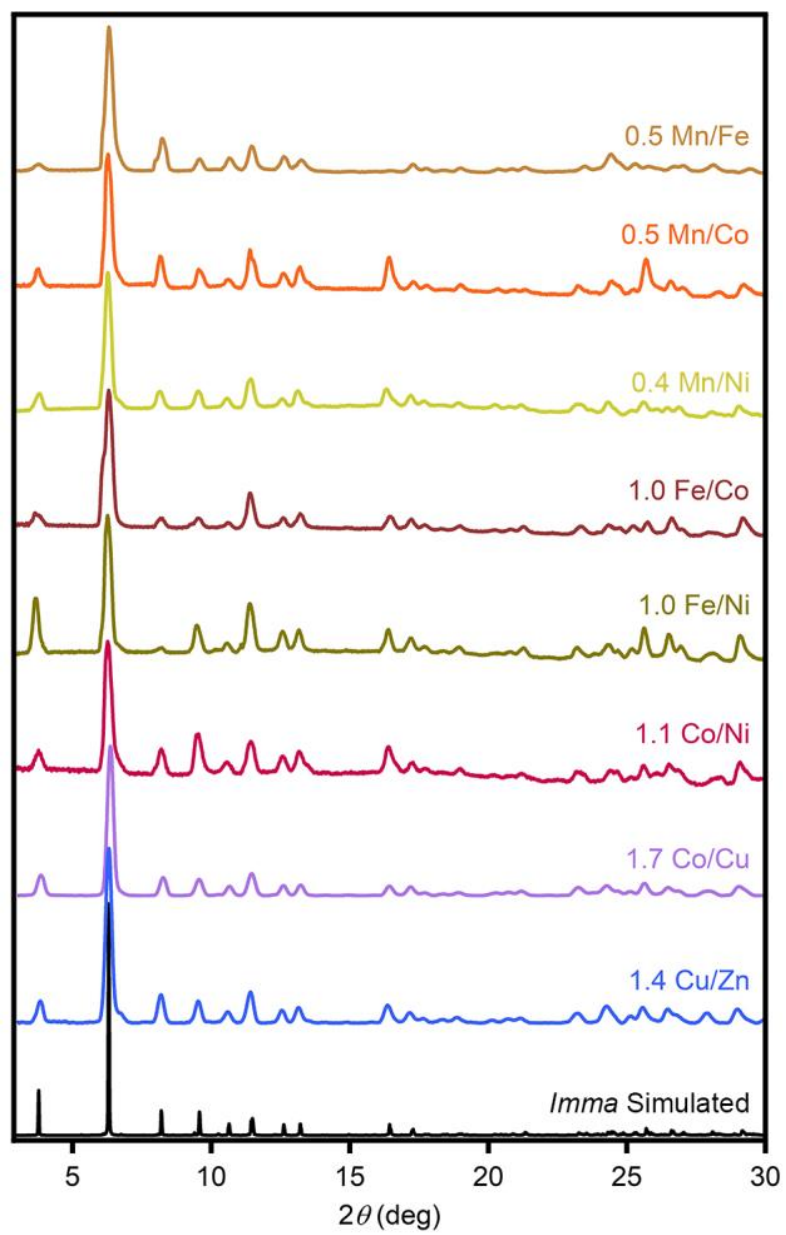

Figure S8. Powder X-ray diffraction patterns for frameworks with mixed-metal bridging-units. Mol ratios are (top to bottom) $0.5 \mathrm{Mn} / \mathrm{Fe}, 0.5 \mathrm{Mn} / \mathrm{Co}, 0.4 \mathrm{Mn} / \mathrm{Ni}$, 1.0 $\mathrm{Fe} / \mathrm{Co}, 1.0 \mathrm{Fe} / \mathrm{Ni}, 1.1 \mathrm{Co} / \mathrm{Ni}, 1.7 \mathrm{Co} / \mathrm{Cu}$ and $1.4 \mathrm{Cu} / \mathrm{Zn}$.

Table S3. Comparison of nominal and experimental ratios in frameworks with mixed-metal bridging-units.

\begin{tabular}{ccc} 
Mixture & $\begin{array}{c}\text { Nominal } \\
\text { Ratio }\end{array}$ & $\begin{array}{c}\text { Experimental } \\
\text { Ratio }\end{array}$ \\
\hline $\mathrm{Mn} / \mathrm{Fe}$ & 1.0 & 0.48 \\
$\mathrm{Mn} / \mathrm{Co}$ & 1.0 & 0.50 \\
$\mathrm{Mn} / \mathrm{Ni}$ & 1.0 & 0.43 \\
$\mathrm{Fe} / \mathrm{Co}$ & 1.0 & 1.0 \\
$\mathrm{Fe} / \mathrm{Ni}$ & 1.0 & 1.0 \\
$\mathrm{Co} / \mathrm{Ni}$ & 1.0 & 1.1 \\
$\mathrm{Co} / \mathrm{Cu}$ & 0.33 & $1.7(63 \%)$ \\
$\mathrm{Co} / \mathrm{Cu}$ & 0.11 & $0.2(17 \%)$ \\
$\mathrm{Cu} / \mathrm{Zn}$ & 0.25 & 1.4
\end{tabular}




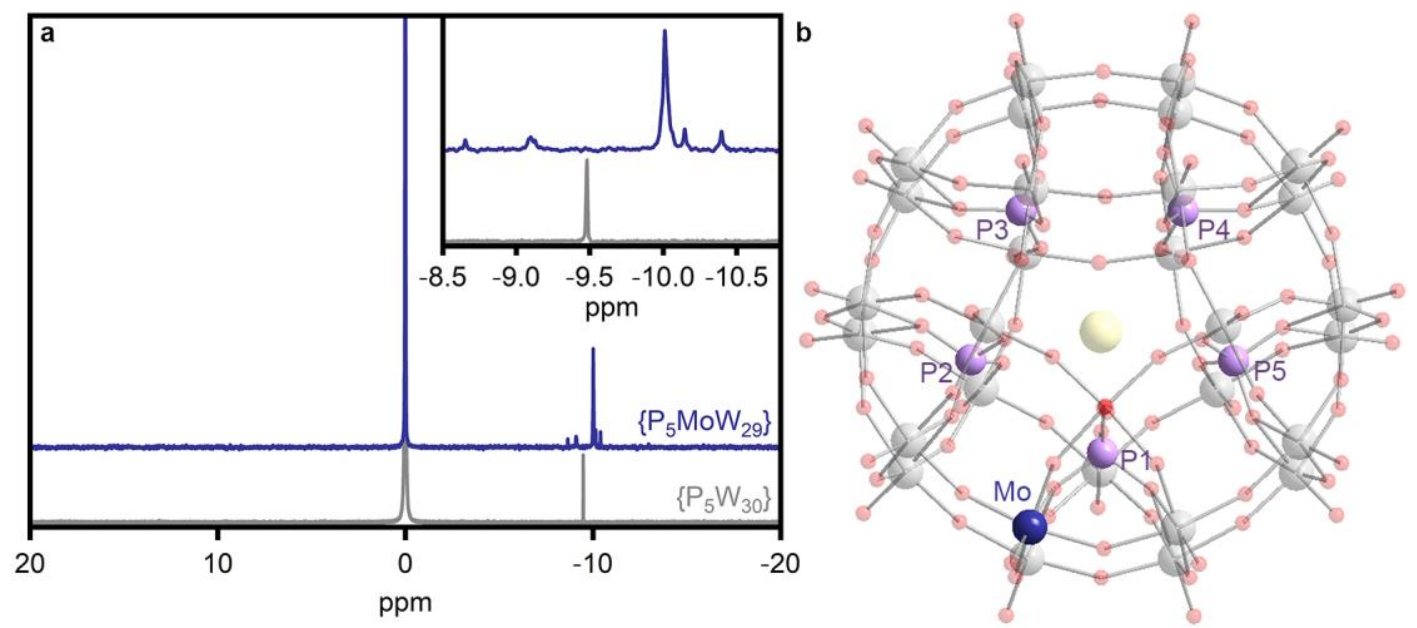

Figure S9. (a) ${ }^{31} \mathrm{P}-\mathrm{NMR}$ spectrum of the Mo-doped cluster, $\mathrm{K}_{14}\left[\mathrm{NaP}_{5} \mathrm{MoW}_{9} \mathrm{O}_{110}\right] \cdot 15 \mathrm{H}_{2} \mathrm{O}\left(\left\{\mathrm{P}_{5} \mathrm{MoW}_{29}\right\}\right.$, blue, top) compared to that of the undoped cluster $\left(\left\{\mathrm{P}_{5} \mathrm{~W}_{30}\right\}\right.$, gray, bottom). The emergence of $5 \mathrm{P}$ peaks suggests that the Mo is doped into the belt of the cluster, yielding 5 distinct $\mathrm{P}$ environments. (b) Crystal structure of the cluster showing the 5 distinct $\mathrm{P}$ environments formed upon Mo-doping.

Table S4. Crystallographic parameters of Co- and Fe-bridged $\left[\mathrm{NaP}_{5} \mathrm{MoW}_{29} \mathrm{O}_{110}\right]^{14-}$

\begin{tabular}{lcc} 
Bridge & Fe-Mo & Co-Mo \\
\hline Empirical formula & $\mathrm{Fe}_{3.4 \mathrm{~K}_{3.9} \mathrm{LiNaO}_{126.7} \mathrm{P}_{5} \mathrm{MoW}_{29}}$ & $\mathrm{Co}_{.7} \mathrm{~K}_{3.1} \mathrm{Li}_{0.8} \mathrm{Na}_{1.3} \mathrm{O}_{131.8} \mathrm{P}_{5} \mathrm{MoW}_{29}$ \\
Formula weight & 7980.10 & 8066.07 \\
Crystal size $\left(\mathrm{mm}^{3}\right)$ & $0.042 \times 0.031 \times 0.018$ & $0.061 \times 0.045 \times 0.025$ \\
$a(\AA)$ & $52.361(3)$ & $52.118(3)$ \\
$b(\AA)$ & $21.6772(12)$ & $21.5870(12)$ \\
$c(\AA)$ & $26.1817(16)$ & $26.1688(16)$ \\
Volume $\left(\AA^{3}\right)$ & $29717(3)$ & $29442(3)$ \\
$Z$ & 8 & 8 \\
$\rho_{\text {calc }}\left(\mathrm{g} / \mathrm{cm}^{3}\right)$ & 3.567 & 3.639 \\
$\mu\left(\mathrm{mm}^{-1}\right)$ & 23.005 & 23.286 \\
Goodness-of-fit on $\mathrm{F}^{2}$ & 1.175 & 1.137 \\
Reflections collected & 114401 & 113503 \\
Independent reflections & 16393 & 15645 \\
$R 1$ & 0.0831 & 0.0614 \\
$w R$ & 0.1798 & 0.1375 \\
Crystal system & orthorhombic & orthorhombic \\
Space group & Imma & Imma \\
Average M-O $\mathrm{O}_{\text {cluster }}$ & 2.06 & 2.04 \\
distance $(\AA)$ & 1955443 & 1955442 \\
CSD \# & &
\end{tabular}

Radiation: $\operatorname{MoK}_{\alpha}(\lambda=0.71073)$

Temperature: $100 \mathrm{~K}$ 


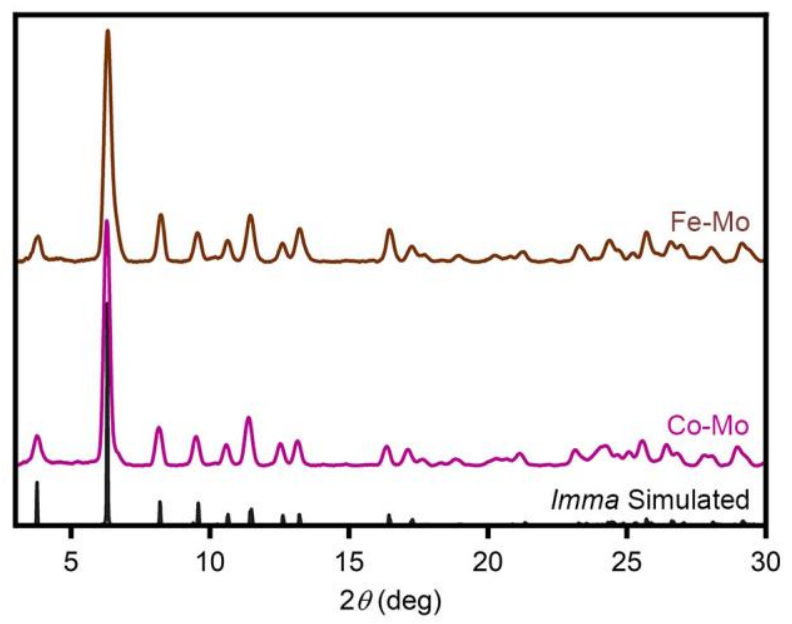

Figure S10. Powder X-ray diffraction patterns of Co- (Co-Mo, pink) and Febridged (Fe-Mo, brown) $\left\{\mathrm{P}_{5} \mathrm{MoW}_{29}\right\}$ compared to the simulated Imma pattern (black).

Table S5. Individual conductivities $(\mu \mathrm{S} / \mathrm{cm})$ of Co-bridged, Fe-bridged and unbridged $\left\{\mathrm{P}_{5} \mathrm{MoW}_{29}\right\}$. Numbers in parentheses are the \% humidity at which each measurement was performed.

\begin{tabular}{rccc} 
& Fe-Mo & Co-Mo & $\left\{\mathrm{P}_{5} \mathrm{MoW}_{29}\right\}$ \\
\cline { 2 - 4 } & 82.0 & 19.3 & 0.64 \\
$(61 \%)$ & $(52 \%)$ & $(50 \%)$ \\
& 60.2 & 12.6 & 0.07 \\
& $(61 \%)$ & $(52 \%)$ & $(50 \%)$ \\
& 64.3 & 15.5 & 0.75 \\
Average & $(60 \%)$ & $(52 \%)$ & $(50 \%)$ \\
Standard deviation & 10 & $\mathbf{1 6}$ & $\mathbf{0 . 5}$ \\
Stevis & 3 & 0.4
\end{tabular}




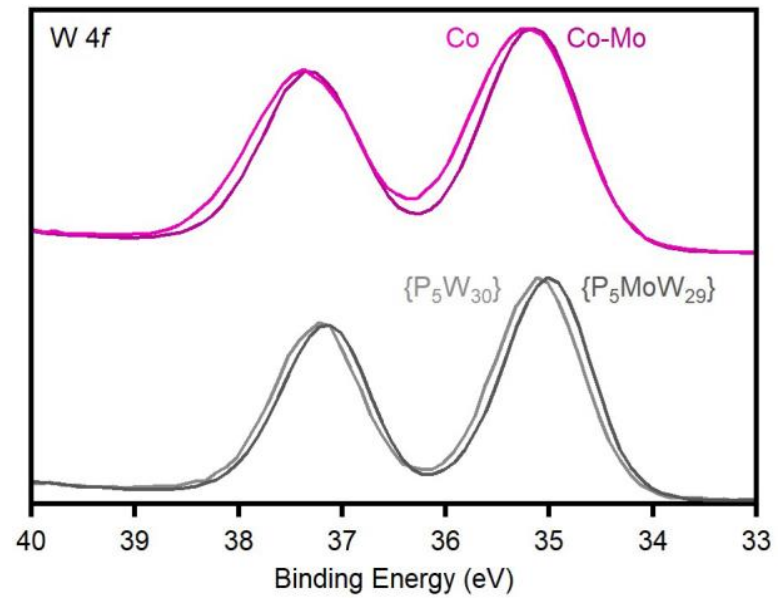

Figure S11. X-ray photoelectron spectra of Co-bridged $\left\{\mathrm{P}_{5} \mathrm{~W}_{30}\right\}$ and $\left\{\mathrm{P}_{5} \mathrm{MoW}_{29}\right\}$ (Co and Co-Mo, respectively; top, pink) and of unbridged $\left\{\mathrm{P}_{5} \mathrm{~W}_{30}\right\}$ and $\left\{\mathrm{P}_{5} \mathrm{MoW}_{30}\right\}$ (bottom, gray). In both cases, inclusion of Mo shifts the $\mathrm{W} 4 f$ peaks to lower binding energy.

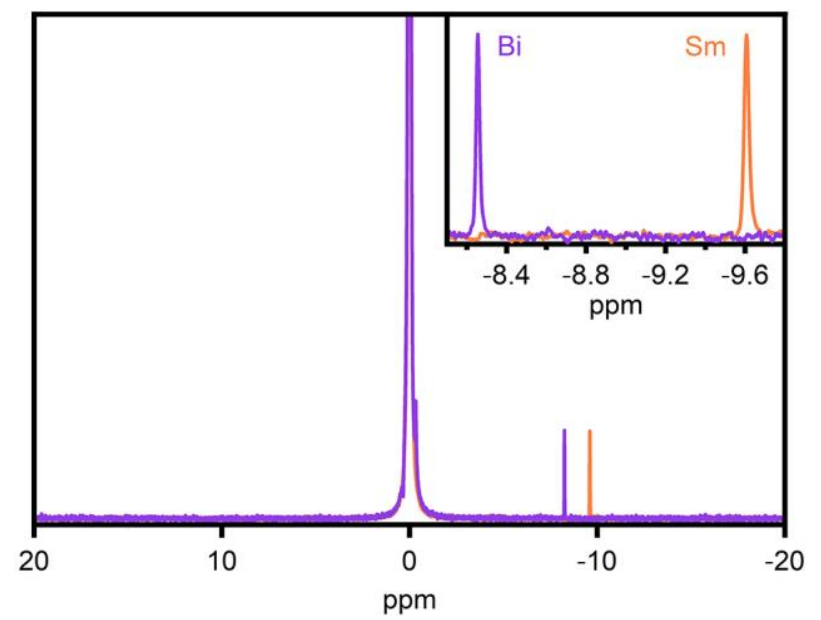

Figure S12. ${ }^{31} \mathrm{P}$ NMR spectra of $\mathrm{K}_{12}\left[\mathrm{SmP}_{5} \mathrm{~W}_{30} \mathrm{O}_{110}\right] \cdot 15 \mathrm{H}_{2} \mathrm{O} \quad(\mathrm{Sm})$ and $\mathrm{K}_{12}\left[\mathrm{BiP}_{5} \mathrm{~W}_{30} \mathrm{O}_{110}\right] \cdot 15 \mathrm{H}_{2} \mathrm{O}(\mathrm{Bi})$. 
Table S6. Crystallographic parameters of Co-bridged $\left[\mathrm{ZP}_{5} \mathrm{MoW}_{29} \mathrm{O}_{110}\right]^{12-}\left(\mathrm{Z}^{3+}=\mathrm{Sm}^{3+}, \mathrm{Bi}^{3+}\right)$

\begin{tabular}{lcc} 
Central ion & Co-Sm & Co-Bi \\
\hline Empirical formula & $\mathrm{Co}_{3 .} \mathrm{K}_{1.8} \mathrm{LiO}_{125.1} \mathrm{SmP}_{5} \mathrm{~W}_{30}$ & $\mathrm{Co}_{3.5} \mathrm{~K}_{3.4} \mathrm{Li}_{0.7} \mathrm{O}_{131.1} \mathrm{BiP}_{5} \mathrm{~W}_{30}$ \\
Formula weight & 8115.06 & 8322.14 \\
Crystal size $\left(\mathrm{mm}^{3}\right)$ & $0.071 \times 0.057 \times 0.043$ & $0.050 \times 0.046 \times 0.032$ \\
$a(\AA)$ & $21.6853(16)$ & $21.6852(16)$ \\
$b(\AA)$ & $79.455(6)$ & $79.711(6)$ \\
$c(\AA)$ & $26.2348(19)$ & $26.2481(19)$ \\
Volume $\left(\AA^{3}\right)$ & $45202(6)$ & $45371(6)$ \\
$Z$ & 12 & 12 \\
$\rho_{\text {calc }}\left(\mathrm{g} / \mathrm{cm}^{3}\right)$ & 3.577 & 3.659 \\
$\mu\left(\mathrm{mm}^{-1}\right)$ & 23.766 & 24.545 \\
Goodness-of-fit on $\mathrm{F}^{2}$ & 1.169 & 1.15 \\
Reflections collected & 170117 & 336427 \\
Independent reflections & 24846 & 22491 \\
$R 1$ & 0.0900 & 0.0644 \\
$w R$ & 0.1930 & 0.1431 \\
Crystal system & orthorhombic & orthorhombic \\
Space group & $C m m e$ & $2.03 m e$ \\
Average M-O $\mathrm{O}_{\text {cluster distance }(\AA)}$ & 2.03 & 1955453 \\
ICSD \# & 1955452 &
\end{tabular}

Radiation: $\operatorname{MoK}_{\alpha}(\lambda=0.71073)$

Temperature: $100 \mathrm{~K}$ 
Table S7. Cluster connections in Co-bridged $\left[\mathrm{ZP}_{5} \mathrm{MoW}_{29} \mathrm{O}_{110}\right]^{12-}\left(\mathrm{Z}^{3+}=\mathrm{Sm}^{3+}, \mathrm{Bi}^{3+}\right)$. Labels are analogous to those of the Imma assembly. ${ }^{1}$

\begin{tabular}{|c|c|c|c|}
\hline \multirow[t]{2}{*}{ Label } & \multirow{2}{*}{ Connectivity } & \multicolumn{2}{|c|}{$\begin{array}{c}\text { Occupancy } \\
\text { Bond Distances }\end{array}$} \\
\hline & & $\mathrm{Sm}$ & $\mathrm{Bi}$ \\
\hline 1 & $\begin{array}{c}\text { Belt of cluster } 1 \text { to } \\
\text { cap of cluster } 2 \text { in the } \\
\text { ab plane }\end{array}$ & $\begin{array}{c}0.50 \\
2.01(3) \\
2.06(2)\end{array}$ & $\begin{array}{c}0.49 \\
2.01(7) \\
2.04(5)\end{array}$ \\
\hline \multirow{2}{*}{2} & \multirow{2}{*}{$\begin{array}{l}\text { Cluster } 1 \text { belt-to-cap } \\
\text { in the ac plane }\end{array}$} & $\begin{array}{l}\text { a: } 0.41 \\
2.01(2) \\
2.02(3)\end{array}$ & $\begin{array}{l}\text { a: } 0.45 \\
1.99(3) \\
2.00(2)\end{array}$ \\
\hline & & $\begin{array}{l}\text { b: } 0.35 \\
2.08(3) \\
2.07(3)\end{array}$ & $\begin{array}{l}\text { b: } 0.31 \\
2.11(2) \\
2.03(2)\end{array}$ \\
\hline 3 & $\begin{array}{l}\text { No connection - only } \\
\text { coordinated by } \\
\text { cluster } 1\end{array}$ & $\begin{array}{c}0.11 \\
2.18(6) \\
2.08(8)\end{array}$ & $\begin{array}{c}0.21 \\
2.23(3) \\
2.06(5)\end{array}$ \\
\hline 4 & $\begin{array}{c}\text { Cap of cluster } 1 \text { to } \\
\text { belt of cluster } 2 \text { in the } \\
\text { ab plane }\end{array}$ & $\begin{array}{c}0.50 \\
2.01(2) \\
1.97(2)\end{array}$ & $\begin{array}{c}0.46 \\
2.00(7) \\
1.97(2)\end{array}$ \\
\hline 5 & $\begin{array}{l}\text { In pore/charge- } \\
\text { compensating }\end{array}$ & & \\
\hline
\end{tabular}

\section{Experimental Methods}

\section{Chemicals and basic characterization}

Chemicals. Sodium chloride $(\mathrm{NaCl},>99 \%)$, lithium chloride $(\mathrm{LiCl},>99 \%)$, methanol $(\mathrm{MeOH}$, 99.9\%), copper(II) chloride dihydrate $\left(\mathrm{CuCl}_{2} \cdot 2 \mathrm{H}_{2} \mathrm{O},>99 \%\right)$ zinc chloride $\left(\mathrm{ZnCl}_{2},>95 \%\right)$, hydrochloric acid $(\mathrm{HCl})$ and barium sulfate $(\mathrm{BaSO} 4,>99 \%)$ were purchased from Fisher Chemical. Cobalt(II) chloride hexahydrate $\left(\mathrm{CoCl}_{2} \cdot 6 \mathrm{H}_{2} \mathrm{O}, 98 \%\right)$ and bismuth(III) chloride $\left(\mathrm{BiCl}_{3}\right.$, anhydrous, >97\%) were purchased from TCI America. Manganese(II) chloride tetrahydrate $\left(\mathrm{MnCl}_{2} \cdot 4 \mathrm{H}_{2} \mathrm{O},>95 \%\right)$ was purchased from J. T Baker. Iron(II) chloride tetrahydrate $\left(\mathrm{FeCl}_{2} \cdot 4 \mathrm{H}_{2} \mathrm{O}\right.$, $98 \%)$, lithium perchlorate trihydrate $\left(\mathrm{LiClO}_{4} \cdot 3 \mathrm{H}_{2} \mathrm{O},>95 \%\right)$, samarium(III) chloride hydrate $\left(\mathrm{SmCl}_{3} \cdot x \mathrm{H}_{2} \mathrm{O}, 99.9 \%\right)$ and potassium chloride $(\mathrm{KCl}, 99 \%)$ were purchased from Alfa Aesar. Sodium tungstate dihydrate $\left(\mathrm{Na}_{2} \mathrm{WO}_{4} \cdot 2 \mathrm{H}_{2} \mathrm{O}, 95 \%\right)$ and nickel(II) chloride hexahydrate $\left(\mathrm{NiCl}_{2} \cdot 6 \mathrm{H}_{2} \mathrm{O}, 98 \%\right)$ were purchased from Spectrum Chemical. Phosphoric acid $\left(\mathrm{H}_{3} \mathrm{PO}_{4}, 85 \%\right)$ and sodium molybdate dihydrate $\left(\mathrm{Na}_{2} \mathrm{MoO}_{4} \cdot 2 \mathrm{H}_{2} \mathrm{O}, 95 \%\right)$ were purchased from Acros Organics. Zinc acetate $\left(\mathrm{Zn}(\mathrm{OAc})_{2} \cdot 2 \mathrm{H}_{2} \mathrm{O}\right)$ was purchased from Ward's Science. All chemicals were used without further purification.

Characterization. Inductively coupled plasma mass spectrometry (ICP-MS) was collected on a Thermo iCAP RQ ICP-MS. The samples were digested in a 1:1 mixture of $85 \% \mathrm{HNO}_{3}(\mathrm{Optima}$ 
grade, Sigma Aldrich) and $30 \% \mathrm{H}_{2} \mathrm{O}_{2}$ (trace metal grade, Sigma Aldrich) with sonication for 90 min. Thermogravimetric Analysis (TGA) was collected using a Perkin-Elmer STA 6000 thermogravimetric analyzer under $\mathrm{N}_{2}$ with a heating rate of $5^{\circ} \mathrm{C} / \mathrm{s}$. The percent weight in water was determined by the mass loss from $30{ }^{\circ} \mathrm{C}$ to $400^{\circ} \mathrm{C}$. Infrared spectroscopy (FTIR) was collected using an Agilent Cary 630 ATR-FTIR. ${ }^{31} \mathrm{P}$ NMR was collected on a JEOL ECA $500 \mathrm{MHz}$ spectrometer with $\mathrm{H}_{3} \mathrm{PO}_{4}$ in a capillary as an internal standard. Powder X-ray diffraction patterns were collected on a Bruker K3 Kappa Vantec 500 diffractometer equipped with $\mathrm{Cu} \mathrm{K}_{\alpha}$ radiation $(\lambda=1.54184)$. Diffraction images were merged/integrated in Diffrac.EVA V.4.2.2 (Bruker).

\section{Synthesis}

Synthesis of the Preyssler cluster, $\mathrm{K}_{12.5} \mathrm{Na}_{1.5}\left[\mathrm{NaP}_{5} \mathrm{~W}_{30} \mathrm{O}_{110}\right] \cdot 15 \mathrm{H}_{2} \mathrm{O}\left(\left\{\mathrm{P}_{5} \mathrm{~W}_{30}\right\}\right)$. The Preyssler cluster was synthesized following literature procedures. ${ }^{4} \mathrm{Na}_{2} \mathrm{WO}_{4} \cdot 2 \mathrm{H}_{2} \mathrm{O}(9.90 \mathrm{~g}, 27 \mathrm{mmol}), 85 \%$ $\mathrm{H}_{3} \mathrm{PO}_{4}(7.0 \mathrm{ml})$ and $\mathrm{NaCl}(1.17 \mathrm{~g}, 20 \mathrm{mmol})$ were mixed with deionized water $(9.0 \mathrm{ml})$ in a $43 \mathrm{ml}$ Teflon-lined acid digestion vessel. The reaction mixture was stirred until all reactants were dissolved at room temperature after which the autoclave reactor was placed in an oven at $125{ }^{\circ} \mathrm{C}$ for $20 \mathrm{~h}$. After the solution mixture was cooled to room temperature $\mathrm{KCl}(3.00 \mathrm{~g}, 0.040 \mathrm{~mol})$ was added and stirred for $30 \mathrm{~min}$. The pale-yellow precipitate was collected by filtration. The product was recrystallized 3 times with $10 \mathrm{ml}$ hot DI water. The final white crystals were collected by filtration and dried for $3 \mathrm{~h}$. Yield: $1.8 \mathrm{~g}, 0.21 \mathrm{mmol}, 21 \%$ based on W. Characteristic FTIR bands $\left(\mathrm{cm}^{-1}\right)$ : 3403(s), 1620 (s), 1157 (s), $1075(\mathrm{~m}), 1016$ (w), 904 (s), 704 (s). ${ }^{31}$ P NMR: $\delta$-9.495 ppm (s).

Synthesis of Mo-doped cluster, $\mathrm{K}_{14}\left[\mathrm{NaP}_{5} \mathrm{MoW}_{9} \mathrm{O}_{110}\right] \cdot 15 \mathrm{H}_{2} \mathrm{O}\left(\left\{\mathrm{P}_{5} \mathrm{MoW} \mathrm{W}_{29}\right\}\right)$. DI water (9.0 ml), $\mathrm{Na}_{2} \mathrm{WO}_{4} \cdot 2 \mathrm{H}_{2} \mathrm{O}(9.57 \mathrm{~g}, 26 \mathrm{mmol}), 85 \% \mathrm{H}_{3} \mathrm{PO}_{4}(7.0 \mathrm{ml}), \mathrm{Na}_{2} \mathrm{MoO}_{4} \cdot 2 \mathrm{H}_{2} \mathrm{O}(0.484 \mathrm{~g}, 2 \mathrm{mmol})$ and $\mathrm{NaCl}(1.17 \mathrm{~g}, 20 \mathrm{mmol})$ were added to a 43-ml Teflon-lined acid digestion vessel. The reaction mixture was stirred for $5 \mathrm{~min}$ at room temperature, followed by heating in an oven at $125{ }^{\circ} \mathrm{C}$ for $20 \mathrm{~h}$. After the reaction cooled to room temperature, $\mathrm{KCl}(3.00 \mathrm{~g}, 0.040 \mathrm{~mol})$ was added and the solution stirred for $30 \mathrm{~min}$. The yellow precipitate was collected by filtration. The yellow crystals were dissolved in $10 \mathrm{ml}$ of hot deionized water and left to recrystallize overnight at $20{ }^{\circ} \mathrm{C}$. The final yellow crystals were collected via filtration and dried for $5 \mathrm{~h}$. Mo-doping was confirmed by ${ }^{31}$ P-NMR (Figure S9) and elemental analysis. Specifically, ICP-MS yields one Mo per $\left\{\mathrm{P}_{5} \mathrm{~W}_{30}\right\}$ and ${ }^{31} \mathrm{P}-\mathrm{NMR}$ reveals the emergence of 5 distinct $\mathrm{P}$ environments (Figure S9). Substitution of Mo into a belt site would break the pseudo- $\mathrm{D}_{5 \mathrm{~h}}$ symmetry of $\left\{\mathrm{P}_{5} \mathrm{~W}_{30}\right\}$ (Figure $\mathrm{S} 9 \mathrm{~b}$ ) and is thus consistent with the observed ${ }^{31} \mathrm{P}-\mathrm{NMR}$ spectrum. Based on these results, we assigned 5\% Mo (1/20 belt sites) on each belt-W in the crystal structure solution. Yield: $2.4 \mathrm{~g}, 0.28 \mathrm{mmol}, 28 \%$ based on W. Characteristic FTIR bands (cm $\left.{ }^{-1}\right)$ : 1616 (s), 1159 (s), $1075(\mathrm{~m}), 1012$ (w), 907 (s), 703 (s).

Synthesis of $\mathrm{K}_{12}\left[\mathrm{ZP}_{5} \mathrm{~W}_{30} \mathrm{O}_{110}\right] \cdot 15 \mathrm{H}_{2} \mathrm{O}\left(\mathrm{Z}^{3+}=\mathrm{Sm}^{3+}, \mathrm{Bi}^{3+}\right)$. Exchange of the central ion was performed following established procedures. ${ }^{5} \mathrm{~K}_{12.5} \mathrm{Na}_{1.5}\left[\mathrm{NaP}_{5} \mathrm{~W}_{30} \mathrm{O}_{110}\right] \cdot 15 \mathrm{H}_{2} \mathrm{O}(1.00 \mathrm{~g}, 0.125$ mmol) and metal salt $\left(\mathrm{MCl}_{3}, \mathrm{M}^{3+}=\mathrm{Bi}^{3+}, \mathrm{Sm}^{3+}, 0.250 \mathrm{mmol}\right)$ were dissolved in DI water (3 ml) and stirred for 15-20 min. The mixture was transferred to a 43-ml Teflon-lined acid digestion vessel and heated at $170{ }^{\circ} \mathrm{C}$ for $20 \mathrm{~h}$. After the solution cooled to room-temperature, $\mathrm{KCl}(3.00 \mathrm{~g}, 0.040$ mol) was added and the white precipitate was collected via filtration. Complete cavity exchange was verified by ${ }^{31} \mathrm{P}$ NMR (Figure S12).

Synthesis of Mn-bridged frameworks, $\mathrm{H}_{4.2} \mathrm{~K}_{1.7} \mathrm{Li}_{0.3} \mathrm{Na}_{1.2}\left[\mathrm{Mn}_{3.3} \mathrm{NaP}_{5} \mathrm{~W}_{30} \mathrm{O}_{110}\right] \cdot 34 \mathrm{H}_{2} \mathrm{O}(\mathrm{Mn})$. Mnbridged frameworks were synthesized following our previously published procedure for Cobridged frameworks. ${ }^{1} \mathrm{MnCl}_{2} \cdot 4 \mathrm{H}_{2} \mathrm{O}(130 \mathrm{mg}, 0.657 \mathrm{mmol}), \mathrm{K}_{12.5} \mathrm{Na}_{1.5}\left[\mathrm{NaP}_{5} \mathrm{~W}_{30} \mathrm{O}_{110}\right] \cdot 15 \mathrm{H}_{2} \mathrm{O}$ $(130.0 \mathrm{mg}, 0.015 \mathrm{mmol})$ and aqueous $\mathrm{LiCl}(7.0 \mathrm{ml}, 1 \mathrm{M}, \mathrm{pH} 1.0$, adjusted by $4 \mathrm{M} \mathrm{HCl})$ were added 
to a $25-\mathrm{ml}$ round-bottom flask equipped with a reflux condenser. The resulting light pink solution was heated at $90{ }^{\circ} \mathrm{C}$ with stirring at for $20 \mathrm{~h}$. The solution was then transferred to a $20-\mathrm{ml}$ vial and concentrated by heating at $80{ }^{\circ} \mathrm{C}$ to a volume of $\sim 1 \mathrm{ml}(\sim 3 \mathrm{~h})$. The reaction was performed $2-5$ times and the vials placed into a sealed $60-\mathrm{ml}$ jar containing $20 \mathrm{ml} \mathrm{MeOH}$. Pale yellow crystals were observed within one week and collected after two weeks. Crystals were washed 6 times with $10 \mathrm{ml} \mathrm{MeOH}$ to remove any chloride salt impurities, as evidenced by the loss of $\mathrm{Cl}$ absorption in the energy dispersive X-ray spectra. Yield (one vial): $95 \mathrm{mg}, 0.011 \mathrm{mmol}, 76 \%$ based on $\left\{\mathrm{P}_{5} \mathrm{~W}_{30}\right\}$. ICP-MS analysis: $\mathrm{H}_{4.2} \mathrm{~K}_{1.7} \mathrm{Li}_{0.3} \mathrm{Na}_{1.2}\left[\mathrm{Mn}_{3.3} \mathrm{NaP}_{5} \mathrm{~W}_{30} \mathrm{O}_{110}\right] \cdot 34 \mathrm{H}_{2} \mathrm{O}, \quad \mathrm{MW} \quad 8346.463 \mathrm{~g} / \mathrm{mol}$. Calculated (experimental) \% mass: Li 0.025 (0.026), K 0.79 (0.82), Mn 2.17 (2.15), Na 0.61 (0.61), P 1.86 (1.86), W 66.08 (65.83). TGA for water loss from $30{ }^{\circ} \mathrm{C}$ to $400{ }^{\circ} \mathrm{C}$, calculated (experimental): 7.32 (7.32)\%.

Synthesis of Fe-bridged frameworks, $\mathrm{H}_{2 .}{ }_{2} \mathrm{LiNa0.3} \mathrm{K}_{2.8} \mathrm{Fe}_{3.5}\left[\mathrm{NaP}_{5} \mathrm{~W}_{30} \mathrm{O}_{110}\right] \cdot 32.5 \mathrm{H}_{2} \mathrm{O}(\mathrm{Fe}) . \mathrm{Fe}-$ bridged frameworks were synthesized following the procedure for Mn-bridged frameworks, using $\mathrm{FeCl}_{2} \cdot 4 \mathrm{H}_{2} \mathrm{O}$ (130 mg, $0.654 \mathrm{mmol}$ ), which yielded a pale yellow solution. Dark brown crystals were observed after one week and collected after two weeks. Yield (one vial): $74 \mathrm{mg}, 0.0089$ mmol, 59\% based on $\left\{\mathrm{P}_{5} \mathrm{~W}_{30}\right\}$. ICP-MS analysis: $\mathrm{H}_{2 .}{ }_{2} \mathrm{LiNa} 0.3 \mathrm{~K}_{2.8} \mathrm{Fe}_{3.5}\left[\mathrm{NaP}_{5} \mathrm{~W}_{30} \mathrm{O}_{110}\right] \cdot 32.5 \mathrm{H}_{2} \mathrm{O}$, MW $8359.395 \mathrm{~g} / \mathrm{mol}$. Calculated (experimental) \% mass: Li 0.083(0.084), K 1.31 (1.30), Fe 2.34 (2.37), Na $0.36(0.35), \mathrm{P} 1.85$ (1.85), W 65.98 (65.51). TGA for water loss from $30{ }^{\circ} \mathrm{C}$ to $400{ }^{\circ} \mathrm{C}$, calculated (experimental): 7.00 (7.01) \%.

Synthesis of Co-bridged frameworks, $\mathrm{HK}_{3.5} \mathrm{Li}_{1.5}\left[\mathrm{Co}_{4} \mathrm{NaP}_{5} \mathrm{~W}_{30} \mathrm{O}_{110}\right] \cdot 37 \mathrm{H}_{2} \mathrm{O}$ (Co). Co-bridged frameworks have been reported previously ${ }^{1}$ and were synthesized following the procedure for $\mathrm{Mn}$ bridged frameworks, using $\mathrm{CoCl}_{2} \cdot 6 \mathrm{H}_{2} \mathrm{O}(156 \mathrm{mg}, 0.656 \mathrm{mmol})$, which yielded a dark blue solution. Pink crystals were observed after one day and collected after one week. Yield (one vial): $114 \mathrm{mg}$, $0.0013 \mathrm{mmol}, 89 \%$ based on $\left\{\mathrm{P}_{5} \mathrm{~W}_{30}\right\}$.

Synthesis of $\mathrm{Ni}$-bridged frameworks, $\mathrm{H}_{5.7 \mathrm{Lio.6}} \mathrm{Na}_{3.3} \mathrm{~K}_{1.4}\left[\mathrm{Ni}_{3} \mathrm{NaP}_{5} \mathrm{~W}_{30} \mathrm{O}_{110]} \cdot 41 \mathrm{H}_{2} \mathrm{O}\right.$ ( $\mathrm{Ni}$ ). Nibridged frameworks were synthesized following the procedure for Mn-bridged frameworks, using $\mathrm{NiCl}_{2} \cdot 6 \mathrm{H}_{2} \mathrm{O}$ (156 mg, $0.656 \mathrm{mmol}$ ), which yielded a green solution. Pale green crystals were observed after one week and collected after two weeks. Yield: $115 \mathrm{mg}, 0.014 \mathrm{mmol}, 91 \%$ based on $\left\{\mathrm{P}_{5} \mathrm{~W}_{30}\right\}$. ICP-MS analysis: $\mathrm{H}_{5.7} \mathrm{Li}_{0.6} \mathrm{Na}_{0.3} \mathrm{~K}_{1.4}\left[\mathrm{Ni}_{3} \mathrm{NaP}_{5} \mathrm{~W}_{30} \mathrm{O}_{110}\right] \cdot 41 \mathrm{H}_{2} \mathrm{O}, \mathrm{MW} 8438.449 \mathrm{~g} / \mathrm{mol}$. Calculated (experimental) \% mass: Li 0.049 (0.047), K 0.64 (0.65), Ni 2.09 (2.07), Na 0.35 (0.36), P 1.83 (1.76), W 65.36 (65.36). TGA for water loss from $30{ }^{\circ} \mathrm{C}$ to $400{ }^{\circ} \mathrm{C}$, calculated (experimental): $8.75(8.80) \%$.

Synthesis of $\mathrm{Cu}$-bridged frameworks, $\mathrm{H}_{4} \mathrm{Na}_{0.8} \mathrm{~K}_{3.2}\left[\mathrm{Cu}_{3} \mathrm{NaP}_{5} \mathrm{~W}_{30} \mathrm{O}_{110}\right] \cdot 35 \mathrm{H}_{2} \mathrm{O}(\mathrm{Cu})$. Cu-bridged frameworks were synthesized following the procedure for Mn-bridged frameworks, using $\mathrm{CuCl}_{2} \cdot 2 \mathrm{H}_{2} \mathrm{O}(104 \mathrm{mg}, 0.610 \mathrm{mmol})$. Additionally, aqueous $\mathrm{LiCl}$ was replaced with aqueous $\mathrm{LiClO}_{4}(2.0 \mathrm{ml}, 0.5 \mathrm{M})$. Pale blue crystals were observed after one week and collected after five weeks. Yield: $92 \mathrm{mg}, 0.011 \mathrm{mmol}, 73 \%$ based on $\left\{\mathrm{P}_{5} \mathrm{~W}_{30}\right\}$. ICP-MS analysis: $\mathrm{H}_{4} \mathrm{Na}_{0.8} \mathrm{~K}_{3.2}$ $\left[\mathrm{Cu}_{3} \mathrm{NaP}_{5} \mathrm{~W}_{30} \mathrm{O}_{110}\right] \cdot 35 \mathrm{H}_{2} \mathrm{O}, \mathrm{MW} 8420.838 \mathrm{~g} / \mathrm{mol}$. Calculated (experimental) \% mass: K 1.48 (1.49), $\mathrm{Cu} 2.26$ (2.27), Na 0.49 (0.49), P 1.84 (1.84), W 65.49 (64.50). TGA for water loss from $30{ }^{\circ} \mathrm{C}$ to $400{ }^{\circ} \mathrm{C}$, calculated (experimental): 7.48 (7.50) \%.

Synthesis of $\mathrm{Zn}$-bridged frameworks, $\mathrm{H}_{3} \mathrm{Na}_{0.7} \mathrm{~K}_{3.3}\left[\mathrm{Zn}_{3.5} \mathrm{NaP}_{5} \mathrm{~W}_{30} \mathrm{O}_{110}\right] \cdot 37.5 \mathrm{H}_{2} \mathrm{O}$ (Zn). Znbridged frameworks were synthesized following the procedure for $\mathrm{Cu}$-bridged frameworks, using $\mathrm{ZnCl}_{2}(82 \mathrm{mg}, 0.602 \mathrm{mmol})$ or $\mathrm{Zn}(\mathrm{OAc})_{2} \cdot 2 \mathrm{H}_{2} \mathrm{O}$ (132 mg, $\left.0.601 \mathrm{mmol}\right)$. Colorless crystals were observed after one week, but changed color with time. Silver-colored crystals were collected after 5 weeks. Yield: $75 \mathrm{mg}, 0.0088 \mathrm{mmol}, 59 \%$ based on $\left\{\mathrm{P}_{5} \mathrm{~W}_{30}\right\}$. ICP-MS analysis: $\mathrm{H}_{3} \mathrm{Na}_{0.7} \mathrm{~K}_{3.3}$ $\left[\mathrm{Zn}_{3.5} \mathrm{NaP}_{5} \mathrm{~W}_{30} \mathrm{O}_{110}\right] \cdot 37.5 \mathrm{H}_{2} \mathrm{O}, \mathrm{MW} 8504.725 \mathrm{~g} / \mathrm{mol}$. Calculated (experimental) \% mass: K 1.51 
(1.53), Zn 2.69 (2.69), Na 0.46 (0.46), P 1.82 (1.82), W 64.85 (64.10). TGA for water loss from $30{ }^{\circ} \mathrm{C}$ to $400{ }^{\circ} \mathrm{C}$, calculated (experimental): 7.93 (7.90) \%.

The silver color of the $\mathrm{Zn}$-bridged frameworks, due to $\mathrm{Zn}^{0}$, could be removed via post-synthetic oxidation (Figure S5). Specifically, $1 \mathrm{ml} \mathrm{H}_{2} \mathrm{O}_{2}$ was added to a vial of finely powdered frameworks in the mother liquor and allowed to soak for $\sim 12 \mathrm{~h}$. The crystals were then washed 6 times with 10 $\mathrm{ml} \mathrm{MeOH}$ and collected via filtration. The oxidation was monitored using diffuse reflectance spectroscopy (Figure S5a), which revealed a loss of intensity across the visible after oxidation. This change from $\mathrm{Zn}^{0}$ to $\mathrm{Zn}^{2+}$ was confirmed with $\mathrm{Zn}$ LMM X-ray photoelectron spectroscopy ${ }^{2-3}$ (Figure S5b). Powder X-ray diffraction (Figure S5c) revealed no change in the crystal structure of the oxidized frameworks.

Synthesis of Co-bridged, Mo-doped frameworks,

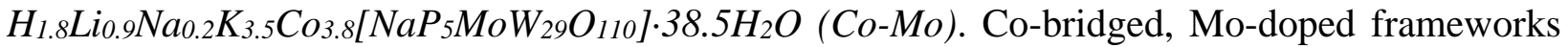
were synthesized following the procedure for Co-bridged frameworks, using $\mathrm{K}_{14}\left[\mathrm{NaP}_{5} \mathrm{MoW}_{29} \mathrm{O}_{110}\right] \cdot 15 \mathrm{H}_{2} \mathrm{O}(130.0 \mathrm{mg}, 0.015 \mathrm{mmol})$. Dark pink crystals were observed after two days and collected after one week. Yield: $93 \mathrm{mg}, 0.11 \mathrm{mmol}, 74 \%$ based on $\left\{\mathrm{P}_{5} \mathrm{MoW}_{29}\right\}$. ICPMS analysis: $\mathrm{H}_{1.8} \mathrm{Li}{ }_{0 .} \mathrm{Na}_{0 .} \mathrm{K}_{3.5} \mathrm{Co}_{3.8}\left[\mathrm{NaP}_{5} \mathrm{MoW}_{29} \mathrm{O}_{110}\right] \cdot 38.5 \mathrm{H}_{2} \mathrm{O}, \mathrm{MW} 8425.934 \mathrm{~g} / \mathrm{mol}$. Calculated (experimental) \% mass: Li 0.070, (0.072) K 1.62 (1.63), Co 2.62 (2.68), Na 0.31 (0.32), P 1.84 (1.78), Mo 1.14 (1.13), W 63.27 (63.27). TGA for water loss from $30{ }^{\circ} \mathrm{C}$ to $400{ }^{\circ} \mathrm{C}$, calculated (experimental): $8.19(8.22) \%$.

Synthesis of Fe-bridged, Mo-doped frameworks, $\mathrm{HLiK}_{3.8} \mathrm{Fe}_{3.4}\left[\mathrm{NaP}_{5} \mathrm{MoW}_{29} \mathrm{O}_{110}\right] \cdot 38 . \mathrm{H}_{2} \mathrm{O}(\mathrm{Fe}$ $\mathrm{Mo}$ ). Fe-bridged, Mo-doped frameworks were synthesized following the procedure for Co-bridged Mo-doped frameworks, using $\mathrm{FeCl}_{2} \cdot 4 \mathrm{H}_{2} \mathrm{O}(130 \mathrm{mg}, 0.654 \mathrm{mmol})$. Black crystals were observed after one week and collected after two weeks. Yield: $87 \mathrm{mg}, 0.10 \mathrm{mmol}, 69 \%$ based on $\left\{\mathrm{P}_{5} \mathrm{MoW}_{29}\right\}$. ICP-MS analysis: $\mathrm{HLiK}_{3.8} \mathrm{Fe}_{3.4}\left[\mathrm{NaP}_{5} \mathrm{MoW}_{29} \mathrm{O}_{110}\right] \cdot 38 . \mathrm{H}_{2} \mathrm{O}$, MW $8395.106 \mathrm{~g} / \mathrm{mol}$. Calculated (experimental) \% mass: Li 0.083 (0.082), K 1.77 (1.77), Fe 2.26 (2.25), Na 0.27 (0.28), P 1.84 (1.84), Mo 1.14 (1.05), W 63.50 (63.50). TGA for water loss from $30{ }^{\circ} \mathrm{C}$ to $400{ }^{\circ} \mathrm{C}$, calculated (experimental): $8.14(8.13) \%$.

Synthesis of $\mathrm{H}_{2} \mathrm{LiK}_{1.8}\left[\mathrm{Co}_{3.6} \mathrm{SmP}_{5} \mathrm{~W}_{30} \mathrm{O}_{110}\right] \cdot 5 \mathrm{OH}_{2} \mathrm{O}(\mathrm{Co}-\mathrm{Sm})$. Preparation was similar to that of Co-bridged frameworks, using $\mathrm{K}_{14}\left[\mathrm{SmP}_{5} \mathrm{~W}_{30} \mathrm{O}_{110}\right] \cdot 15 \mathrm{H}_{2} \mathrm{O}(130 \mathrm{mg}, 0.015 \mathrm{mmol})$. Pink crystals were observed after two weeks and collected after 4 weeks. Yield: $67 \mathrm{mg}, 0.0076 \mathrm{mmol}, 51 \%$ based on $\left[\mathrm{SmP}_{5} \mathrm{~W}_{30} \mathrm{O}_{110}\right]^{12-}$. ICP-MS analysis: $\mathrm{H}_{2} \mathrm{LiK}_{1.8}\left[\mathrm{Co}_{3.6} \mathrm{SmP}_{5} \mathrm{~W}_{30} \mathrm{O}_{110}\right] \cdot 37 \mathrm{H}_{2} \mathrm{O}$, MW $8771.651 \mathrm{~g} / \mathrm{mol}$. Calculated (experimental): Li 0.079 (0.082), K 0.80 (0.78), Co 2.42 (2.43), Sm 1.71 (1.72), P $1.76(1.71), \mathrm{W} 62.88$ (62.87). TGA for water loss from $30{ }^{\circ} \mathrm{C}$ to $400{ }^{\circ} \mathrm{C}$, calculated (found): $10.26(10.29) \%$.

Synthesis of $\mathrm{H}_{0.8} \mathrm{Li}_{0.7} \mathrm{~K}_{3.4}\left[\mathrm{CO}_{3.5} \mathrm{BiP}_{5} \mathrm{~W}_{30} \mathrm{O}_{110}\right] \cdot 3 \mathrm{H}_{2} \mathrm{O}(\mathrm{Co}-\mathrm{Bi})$. Preparation was similar to that of Co-bridged frameworks, using $\mathrm{K}_{14}\left[\mathrm{BiP}_{5} \mathrm{~W}_{30} \mathrm{O}_{110}\right] \cdot 15 \mathrm{H}_{2} \mathrm{O}(130 \mathrm{mg}, 0.015 \mathrm{mmol})$. Pink crystals were observed after two weeks and collected after 4 weeks. Yield: $58 \mathrm{mg}, 0.0067 \mathrm{mmol}, 45 \%$ based on $\left[\mathrm{BiP}_{5} \mathrm{~W}_{30} \mathrm{O}_{110}\right]^{12-}$. This structure exhibited disorder in the central ion and our solution contains a $3 \%$ occupied $\mathrm{Bi}$ position in addition to the $\sim 50 \%$ position normally observed in the $\left\{\mathrm{P}_{5} \mathrm{~W}_{30}\right\}$ cavity. Because ${ }^{31} \mathrm{P}-\mathrm{NMR}$ did not reveal any $\left\{\mathrm{P}_{5} \mathrm{~W}_{30}\right\}$ with $\mathrm{K}^{+}$or $\mathrm{Na}^{+}$as central cavity ions, we assigned this electron density to a low-occupancy disordered $\mathrm{Bi}^{3+}$. ICP-MS analysis: $\mathrm{H}_{0.8} \mathrm{Li}_{0.7} \mathrm{~K}_{3.4}\left[\mathrm{Co}_{3.5} \mathrm{BiP}_{5} \mathrm{~W}_{30} \mathrm{O}_{110}\right] \cdot 37 \mathrm{H}_{2} \mathrm{O}$, MW $8653.696 \mathrm{~g} / \mathrm{mol}$. Calculated (experimental) \% mass: Li 0.060 (0.061), K 1.57 (1.53), Co 2.38 (2.47), Bi 2.41 (2.49), P 1.79 (1.73), W 63.73 (63.73). TGA for water loss from $30{ }^{\circ} \mathrm{C}$ to $400{ }^{\circ} \mathrm{C}$, calculated (experimental): 7.73 (7.73) \% . 


\section{Structural determination}

Single crystal X-ray diffraction studies were performed using a Bruker APEX-II Ultra CCD diffractometer equipped with $M o K_{\alpha}$ radiation $(\lambda=0.71073 \AA)$. Crystals were mounted on a Cryoloop with Fomblin Y oil. Data were collected in a nitrogen gas stream at 100(2) K using $\phi$ and $\varpi$ scans. Crystal-to-detector distance was $45 \mathrm{~mm}$ and exposure time was $10 \mathrm{~s}$ per frame using a scan width of $0.75^{\circ}$. Indexing and unit cell refinement indicated a primitive, orthorhombic lattice. The data were integrated using the Bruker SAINT software program and scaled using the SADABS software program. Solution by direct methods $\left(\right.$ SHELXT $\left.^{6}\right)$ produced a complete phasing model consistent with the proposed structure. All nonhydrogen atoms were refined anisotropically by full-matrix least-squares (SHELXL-2014). ${ }^{6}$

The frameworks contain disorder in the bridging metal ions and in the cap-site $\mathrm{W}$ atoms of one of the clusters. Occupancies of disordered atoms were refined as free variables. To model the disorder, ISOR, EADP and RIGU commands were used. For the disorder in the cap-site W atoms, all Imma structures contained 50/50 occupancy of the two possible sites. For the Cmme structures, FVAR was used to locate and group the disordered $\mathrm{W}$ to match the electron density mapping. These frameworks contained 95/5 and 93/7 occupancies of the two sites for Co-Sm and Co-Bi, respectively. The corresponding disordered $\mathrm{O}$ atoms could not be located due to the low electron density. For highly disordered water molecules, the PLATON routine SQUEEZE ${ }^{7}$ was used to account for the corresponding electrons as a diffuse contribution to the overall scattering without specific atom positions.

In all cases, structures were refined to match the elemental formula determined by ICP-MS, with any remaining negative charge compensated by protons.

\section{Additional characterization}

Diffuse reflectance spectroscopy. Approximately $50 \mathrm{mg}$ framework was finely powdered and mixed 3:1 with $\mathrm{BaSO}_{4}$ using a mortar and pestle. The mixture was loaded into a powder cell holder and spectra were collected using a Cary 5000 equipped with a DRA-2500 internal diffuse reflectance accessory.

Surface area analysis. Nitrogen isotherms were measured by a volumetric method using a Micromeritics ASAP 2020 adsorption analyzer. To a pre-massed sample tube was added a sample of finely powdered Co framework, which was degassed at $105{ }^{\circ} \mathrm{C}$ on the ASAP until the outgas rate was $<5 \mathrm{mmHg}$. After degassing, the tube was massed to determine the mass of framework. Brunauer-Emmett-Teller surface area $\left(\mathrm{m}^{2} / \mathrm{g}\right)$ measurements were performed using a liquid nitrogen bath at $77 \mathrm{~K}$. Isotherms were measured using $99.999 \%$ purity nitrogen (Praxair NI 5.0UM). Oil-free vacuum pumps were used to prevent contamination of sample or feed gases.

$X$-ray photoelectron spectroscopy. XPS measurements were acquired using a Kratos Analytical AXIS Supra surface analysis instrument under $5 \times 10^{-8}$ torr using a monochromatic Al $\mathrm{K}_{\mathrm{a}} \mathrm{X}$-ray source operating at $300 \mathrm{~W}$ with an emission current of $20 \mathrm{~mA}$. Survey spectra were measured with a $1 \mathrm{eV}$ step, dwell-time of $100 \mathrm{~ms}$ and pass energy of $160 \mathrm{eV}$. Detail spectra of $\mathrm{O}$ $1 s, \mathrm{~W} 4 f, \mathrm{Fe} 2 p$, Mo $3 d$ and Zn LMM were averaged over 30 scans with a $0.1 \mathrm{eV}$ step, $100 \mathrm{~ms}$ dwell-time and pass energy of $20 \mathrm{eV}$. Analysis of the XPS spectra was performed using Casaxps software and calibrated spectra based on $\mathrm{O} 1 \mathrm{~s}$ at $530.0 \mathrm{eV}$. After background subtraction using the Shirley routine, spectra were fitted with a convolution of Lorentzian and Gaussian profiles.

Energy-dispersive spectroscopy. EDS measurements were collected on a FEI Quanta FEG 250.

Conductivity measurements. Pressed-pellets were made by loading $\sim 80 \mathrm{mg}$ dried powder into a $7 \mathrm{~mm}$ die (Specac GS03950) and pressing at 2 ton for $1 \mathrm{~h}$. Pellet thicknesses were measured 
using digital calipers. Two gold wires (Alfa Aesar, $0.1 \mathrm{~mm}$ ) were affixed to the pellet in a linear two-wire arrangement using carbon paste (Ted Pella DAG-T-502), which was allowed to dry for $>20$ min prior to data collection. $I-V$ curves were collected on a Gamry 1010E potentiostat using linear sweep voltammetry mode with a scan rate of $100 \mathrm{mV} / \mathrm{s}$ and a step size of $5 \mathrm{mV}$. Resistance $(R)$ values were determined by a linear fit to the $I$ vs $V$ data (where $R=1 /$ slope). Conductivities were calculated as detailed previously. ${ }^{1}$

Conversion of Co-framework to tetrahedral coordination. A sample of finely powdered Co framework $\left(50 \mathrm{mg}\right.$ ) was degassed under vacuum at $90{ }^{\circ} \mathrm{C}$ for $1 \mathrm{~h}$ and brought into a nitrogen-filled glovebox. The evacuated powders were mixed with $\sim 150 \mathrm{mg} \mathrm{BaSO} 4$ and loaded into a powder cell for diffuse reflectance measurements.

\section{References}

1. Turo, M. J.; Chen, L. F.; Moore, C. E.; Schimpf, A. M., $\mathrm{Co}^{2+}-$ Linked $\left[\mathrm{NaP}_{5} \mathrm{~W}_{30} \mathrm{O}_{110}\right]^{14-}$ : A Redox-Active Metal Oxide Framework with High Electron Density. J. Am. Chem. Soc. 2019, $141,4553$.

2. Dake, L. S.; Baer, D. R.; Zachara, J. M., Auger Parameter Measurements of Zinc-Compounds Relevant to Zinc Transport in the Environment. Surf. Interface Anal. 1989, 14, 71.

3. Zuo, J. A.; Erbe, A., Optical and Electronic Properties of Native Zinc Oxide Films on Polycrystalline Zn. Phys. Chem. Chem. Phys. 2010, 12, 11467.

4. Hayashi, A.; Haioka, T.; Takahashi, K.; Bassil, B. S.; Kortz, U.; Sano, T.; Sadakane, M., Cation Effect on Formation of Preyssler-Type 30-Tungsto-5-Phosphate: Enhanced Yield of NaEncapsulated Derivative and Direct Synthesis of $\mathrm{Ca}$ - and Bi-Encapsulated Derivatives. $Z$. Anorg. Allg. Chem. 2015, 641, 2670.

5. Takahashi, K.; Sano, T.; Sadakane, M., Preparation and Characterization of Preyssler-Type Phosphotungstic Acid, $\mathrm{H}_{15-n}\left[\mathrm{P}_{5} \mathrm{~W}_{30} \mathrm{O}_{110} \mathrm{M}^{n+}\right]$, with Different Encapsulated Cations $(\mathrm{M}=\mathrm{Na}$, $\mathrm{Ca}, \mathrm{Bi}, \mathrm{Eu}, \mathrm{Y}$, or Ce), and their Thermal Stability and Acid Catalyst Properties. Z. Anorg. Allg. Chem. 2014, 640, 1314.

6. Sheldrick, G. M., SHELXT - Integrated Space-Group and Crystal-Structure Determination. Acta Crystallogr. A 2015, 71, 3.

7. Spek, A. L., PLATON SQUEEZE: A Tool for the Calculation of the Disordered Solvent Contribution to the Calculated Structure Factors. Acta Crystallogr. C 2015, 71, 9. 